\title{
Erratum to: Efficacy and safety of sitagliptin in elderly patients with type 2 diabetes mellitus and comparison of hypoglycemic action of concomitant medications: a subanalysis of the JAMP study
}

\author{
Noriko Ujihara $^{1} \cdot$ Hiroshi Sakura $^{2} \cdot$ Naotake Hashimoto $^{3} \cdot$ Kazuo Sasamoto $^{4} \cdot$ Hiroshi Ohashi $^{5} \cdot$ Sumiko Hasumi $^{6}$. \\ Tadasu Kasahara $^{7}$ - Osamu Tomonaga ${ }^{8}$ Hideo Nunome ${ }^{9}$ Masashi Honda ${ }^{10} \cdot$ Yasuhiko Iwamoto $^{11} \cdot$ for the JAMP \\ Study Investigators
}

Published online: 14 October 2017

(C) The Japan Diabetes Society 2017

\section{Erratum to: Diabetol Int}

DOI 10.1007/s13340-017-0330-2

In the original publication of the article, a percentage symbol has been included incorrectly in the value of Alcohol consumption (65-74 years) as $45.1 \%$ in Table 1 . The correct version is published with this erratum.

The online version of the original article can be found under doi:10.1007/s13340-017-0330-2.

Noriko Ujihara

ujihara.noriko@twmu.ac.jp

1 Department of Medicine, Diabetes Center, Institute of Geriatrics, Tokyo Women's Medical University, Shibuya Cross Tower 21F, 2-15-1, Shibuya, Shibuya-ku, Tokyo 150-0002, Japan

2 Department of Medicine, Tokyo Women's Medical University, Medical Center East, 2-1-10 Nishiogu, Arakawa-ku, Tokyo 116-8567, Japan

3 Department of Diabetes, Endocrine and Metabolic Diseases, Tokyo Women's Medical University Yachiyo Medical Center, 477-96, Owada-shinden, Yachiyo-shi, Chiba 276-0046, Japan

4 Internal Medicine, Suzuki Clinic, 1F, 2-10-14, Koyasu-machi, Hachioji-shi, Tokyo 192-0904, Japan

5 Internal Medicine, Oyama East Clinic, 1-32-1, Ekihigashi-dori, Oyama-shi, Tochigi 323-0022, Japan

6 Internal Medicine, Nishiyamado-Keiwa Hospital, 3247-1, Kounosu, Naka-shi, Ibaraki 311-0133, Japan
7 Josai Hospital, 2-42-11, Kamiogi, Suginami-ku, Tokyo 167-0043, Japan

8 Diabetes and Lifestyle Center, Tomonaga Clinic, Shinyon curumu Building 9F, 4-2-23, Shinjuku, Shinjuku-ku, Tokyo 160-0022, Japan

9 Diabetes Center, Edogawa Hospital, Medical Plaza Shinozaki, SK Building, 7-15-12, Shinozaki-machi, Edogawa-ku, Tokyo 133-0057, Japan

10 Nishikawa Clinic, 2-16-3, Towa, Adachi-ku, Tokyo 120-0003, Japan

11 Tokyo Women's Medical University/The Institute for Adult Diseases, Asahi Life Foundation, Asahiseimeisunaga Building 2, 3, 4F, 2-2-6, Nihonbashi Bakuro-cho, Chuo-ku, Tokyo 103-0002, Japan 
Table 1 Comparison of patient characteristics between the elderly and non-elderly patients

\begin{tabular}{|c|c|c|c|c|c|c|c|}
\hline & \multicolumn{2}{|c|}{$\geq 75$ years $(n=130)$} & \multicolumn{2}{|c|}{$65-74$ years $(n=189)$} & \multicolumn{2}{|c|}{$<65$ years $(n=332)$} & \multirow[t]{2}{*}{$p$} \\
\hline & $n$ & Mean \pm SD or $\%$ & $n$ & Mean \pm SD or $\%$ & $n$ & Mean \pm SD or $\%$ & \\
\hline Age (years) & 130 & $80.0 \pm 4.7$ & 189 & $69.2 \pm 2.9$ & 332 & $54.5 \pm 7.4$ & $0.000^{*}$ \\
\hline Height $(\mathrm{cm})$ & 127 & $156.2 \pm 9.6$ & 187 & $161.0 \pm 8.6$ & 328 & $166.2 \pm 8.8$ & $0.000 *$ \\
\hline Waist circumference $(\mathrm{cm})$ & 80 & $85.7 \pm 10.5$ & 120 & $87.3 \pm 9.6$ & 193 & $89.9 \pm 12.0$ & $0.009 *$ \\
\hline Duration of diabetes (days) & 111 & $121.4 \pm 94.6$ & 181 & $114.1 \pm 78.4$ & 308 & $95.4 \pm 74.4$ & $0.003 *$ \\
\hline Sex (male) & 67 & 51.5 & 117 & 61.9 & 250 & 75.3 & $0.000 *$ \\
\hline Smoking & 13 & 10.3 & 34 & 18.6 & 96 & 29.8 & $0.000^{*}$ \\
\hline Alcohol consumption & 39 & 31.7 & 82 & 45.1 & 180 & 56.1 & $0.000 *$ \\
\hline Retinopathy & 8 & 6.2 & 8 & 4.2 & 32 & 9.6 & 0.064 \\
\hline Arteriosclerosis obliterans & 6 & 4.6 & 21 & 11.1 & 28 & 8.4 & 0.122 \\
\hline Stroke & 0 & 0.0 & 1 & 0.6 & 0 & 0.0 & 0.288 \\
\hline Myocardial infarction & 7 & 5.4 & 5 & 2.6 & 6 & 1.8 & 0.108 \\
\hline Angina pectoris & 8 & 6.2 & 8 & 4.2 & 11 & 3.3 & 0.387 \\
\hline Cardiac failure & 5 & 3.8 & 2 & 1.1 & 4 & 1.2 & 0.102 \\
\hline Atrial fibrillation & 4 & 3.1 & 5 & 2.6 & 7 & 2.1 & 0.817 \\
\hline Diet/exercise therapy & 49 & 37.7 & 48 & 25.4 & 92 & 27.7 & $0.044 *$ \\
\hline Low dose of glimepiride & 18 & 13.8 & 25 & 13.2 & 29 & 8.7 & 0.153 \\
\hline Medium dose of glimepiride & 9 & 6.9 & 21 & 11.1 & 20 & 6.0 & 0.104 \\
\hline BG & 9 & 6.9 & 25 & 13.2 & 65 & 19.6 & $0.002 *$ \\
\hline TZD & 7 & 5.4 & 12 & 6.3 & 19 & 5.7 & 0.929 \\
\hline$\alpha-\mathrm{GI}$ & 5 & 3.8 & 3 & 1.6 & 10 & 3.0 & 0.446 \\
\hline Multidrug therapy & 33 & 25.4 & 55 & 29.1 & 97 & 29.2 & 0.692 \\
\hline Antihypertensive drug & 81 & 62.3 & 106 & 56.1 & 144 & 43.4 & $0.000^{*}$ \\
\hline Antihyperlipidemic drug & 62 & 47.7 & 94 & 49.7 & 156 & 47.0 & 0.832 \\
\hline Antihyperuricemic drug & 6 & 4.6 & 9 & 4.8 & 16 & 4.8 & 0.996 \\
\hline Antithrombogenic drug & 22 & 16.9 & 44 & 23.3 & 52 & 15.7 & 0.088 \\
\hline
\end{tabular}

$* p<0.05$ 